\title{
SUBSCLERAL SCLERAL BURIAL IN SURGERY OF RETINAL DETACHMENT*
}

\author{
BY \\ GEORGE EL BAYADI \\ Department of Ophthalmology, Faculty of Medicine, Cairo University
}

IN recent years, various operative procedures have been described to bring the diathermy-treated choroid in apposition to the detached retina specially around retinal breaks.

These operations may be divided into two groups: scleral shortening and sclero-choroidal introflexion.

Scleral shortening was revived by Lindner (1933), whose operation is a modification of that of Müller (1903); this involved the removal of a large oval piece of sclera, about $8 \times 20 \mathrm{~mm}$., on the temporal side of the eyeball through a Krönlein exposure, paying no attention to retinal breaks. In Lindner's operation, retinal breaks are treated by diathermy and a long and narrow full-thickness strip of sclera is then excised usually along the temporal half of the equatorial region.

Lindner's full-thickness scleral resection is technically difficult because of the risk of injuring the bulging choroid with attendant vitreous loss and choroidal haemorrhage. Shapland (1951) in England and Paufique (Barut, 1952) in France, described modifications for the full-thickness scleral resection.

The modified operation is the lamellar sclerectomy in which a thin film of the deepest scleral lamellae is left in situ. Scleral shortening operations improved the results of retinal detachment surgery by bringing the choroid nearer to the retina, but the approximation is diffuse and not especially localized to the region of the retinal breaks.

During the last decade two new methods have been introduced which aim at producing localized approximation between the choroid and the retina specially in the region of retinal breaks. In both methods a synthetic implant is stitched to the sclera to produce sclero-choroidal infolding.

Custodis $(1952,1953,1956)$ introduced a polyviol implant which is pressed by sutures against the diathermy-treated surface of the sclera in the region of the retinal breaks. Schepens, Okamura, and Brockhurst (1957, 1958), Okamura, Schepens, and Brockhurst (1959), and Brockhurst, Schepens, and

* Received for publication August 18, 1961. 
Okamura (1958) introduced a polyethylene tube into the bed of a lamellar scleral resection to cause a deep choroidal indentation.

Custodis (1960) found two main difficulties with his operation. The first difficulty is to locate the implant so that the retinal break lies exactly on top of the sclero-choroidal infolding:

"If the retinal break or one end of it is located on the sloping part of the elevation, the re-attachment of the retina and the healing process are questionable".

The second difficulty is the irritating effect of the polyviol implant, which had to be removed in 3-5 per cent. of his cases. Schepens, Okamura, Brockhurst, and Regan (1960) tried the Custodis polyviol implant in 25 eyes and reported that six cases developed an infection in the operated area, necessitating removal of the polyviol and sutures. The seventh case appeared to be sensitive to the polyviol implant.

After long experience in the use of polyethylene tubing in scleral buckling procedures, Schepens and others (1960) reported certain difficulties:

"The hardness of the tube may produce pressure necrosis of the underlying sclera and choroid, and this may create a problem in case a re-operation is necessary. Another difficulty is that the buckling formed by a polyethylene tube is necessarily narrow and a break of large size cannot be adequately covered by the buckling. The narrow scleral buckling tends also to be relatively high because the circling polyethylene tube is often deeply buried. There is so far no convincing evidence that a narrow and very high buckling is more effective in re-attaching the retina than one which is broader and flatter. One of the problems still to be solved is that the lumen of the circling polyethylene tube forms a dead space. If micro-organisms penetrate into the lumen of the tube, it is almost impossible to reach them with systemic antibiotics".

For these reasons Schepens and others (1960) favour silicone implants over polyethylene tubes. Silicone implants are softer and more elastic and can be cut to any required shape, but again Schepens warns that one objection to the implantation of plastic material is its possible carcinogenic action.

For these reasons a technique of subscleral scleral burial has been developed, in which a wide-based area of sclero-choroidal infolding is produced around retinal breaks without the use of a foreign body.

\section{Pre-operative Examination}

The retinal breaks are localized in the usual way. Indirect ophthalmoscopy using a +20 dioptre corrected lens was found more valuable than the usual +13 dioptre condensing lens. With the former lens the field of observation is extremely wide and there is less liability to miss a retinal lesion. Schepens's binocular ophthalmoscope, or Fison's modification, is particularly useful since it provides a brilliant stereoscopic image of the fundus, leaving the observer with both hands 
free to manipulate the condensing lens and make scleral indentations when necessary. After ophthalmoscopy, every case of retinal detachment should be examined biomicroscopically. I prefer my present convex lens (el Bayadi, 1953, 1954, 1961) to the concave lens for the following reasons:

(1) It gives brighter illumination.

(2) It gives a large field of observation.

(3) Highly myopic fundi are easier to examine, a definite advantage in retinal detachment where most eyes are myopic.

(4) The fundus picture is inverted, so the method becomes a continuation of indirect ophthalmoscopy.

\section{Surgical Technique}

(1) Exposure.-The conjunctiva and Tenon's capsule are incised over the region of the retinal breaks. Any muscle in the way is temporarily severed. Vortex veins should be identified and carefully preserved.

(2) Localization.-The exact localization of retinal breaks need close cooperation between surgeon and assistant. The surgeon concentrates the light from a powerful ophthalmoscope on the retinal hole and the assistant should be able to locate the light shining through the sclera. This spot is marked by the assistant with a touch of cautery or diathermy. Next the assistant applies pressure with the tip of a strabismus hook over the previously marked area and now the surgeon, looking through his ophthalmoscope, verifies his localization by finding out whether the indentation is over the area of the retinal break (Fig. 1a).

(3) Diathermy.-A minimal amount of surface diathermy is now applied (Fig. 1b) saving as much as possible the choroid which should be able to absorb the residual subretinal fluid during the first few post-operative days. Excessive diathermy diminishes the absorptive power of the choroid (Fig. 1b).

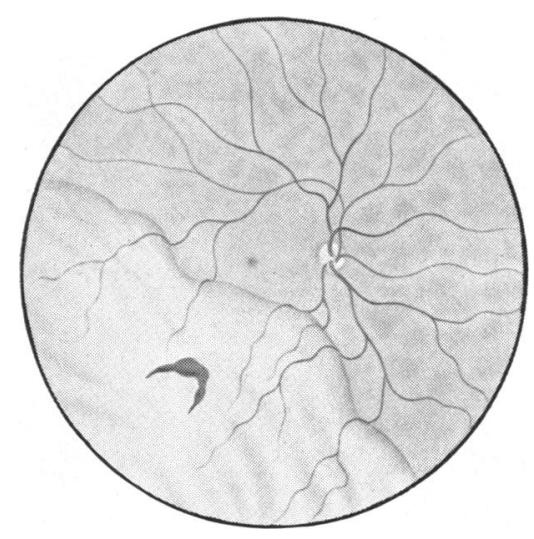

FIG. 1(a).-Localized retinal detachment with peripheral crescentic tear.

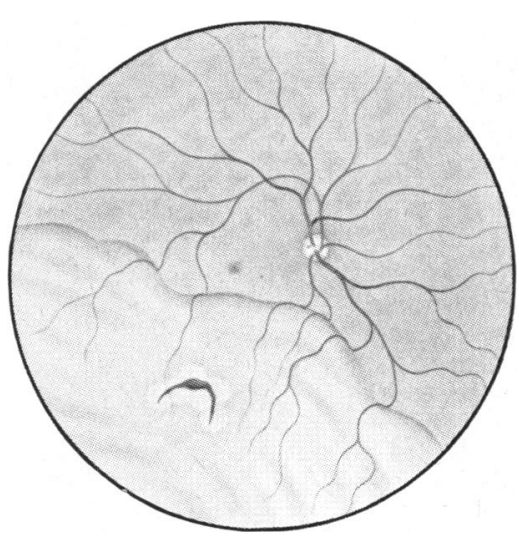

FIG. 1(b).-Diathermy completely surrounds retinal tear.

(4) Subscleral Scleral Burial.-The diathermy-treated area, which overlies the retinal breaks, is now surrounded by a perpendicular scleral incision. If there is 
only one break, or several breaks very near to each other, the diathermy-treated sclera is surrounded by a circular cut preferably made with a corneal trephine (Fig. 2a). The diameter of the trephine used varies from 4 to $7 \mathrm{~mm}$. depending on the size of the retinal break and the degree of retinal elevation. The incision should not go deep to the choroid but the deepest scleral lamellae should be left intact. A trephine with a stop avoids the risk of perforation. The sclera outside the cut is undermined using the tip of a No. 11 Bard-Parker blade or a Desmarres corneal knife (Fig. 2b). The width of the undermined sclera should be slightly more than the radius of the outlined circle. A continuous purse-string suture is now passed through the undermined sclera around the marked scleral disc (Fig. 2c). This suture is tied after evacuation of the subretinal fluid, pushing the scleral disc inwards and burying it under the undermined sclera (Fig. 2d). The underlying choroid is necessarily pushed inward and approximated to the retina around the retinal breaks.

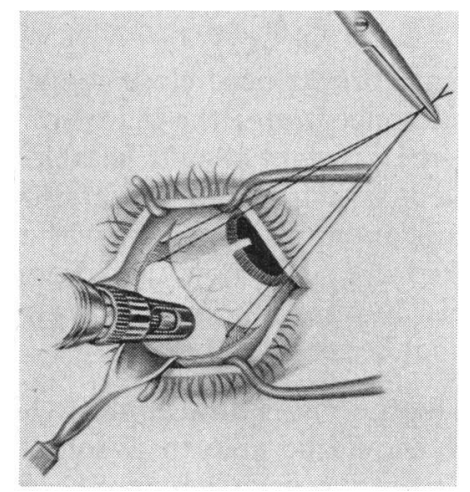

FIG. 2(a).-Diathermy-treated sclera surrounded by circular incision made with corneal trephine.

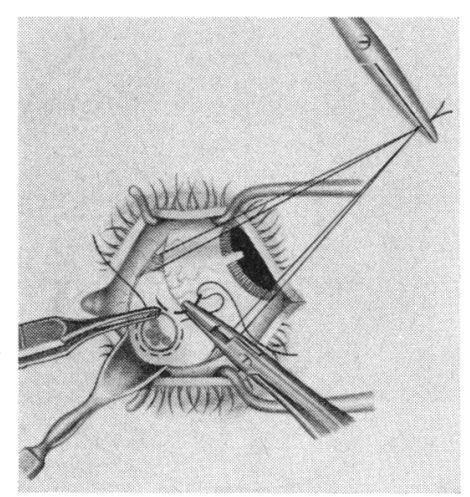

FIG. 2(c).-Purse-string suture inserted in the sclera around the marked scleral disc.

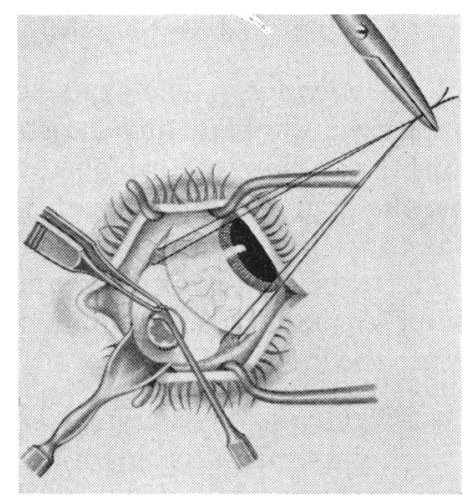

Fig. 2(b).-Sclera around the circular incision undermined using a Desmarres knife.

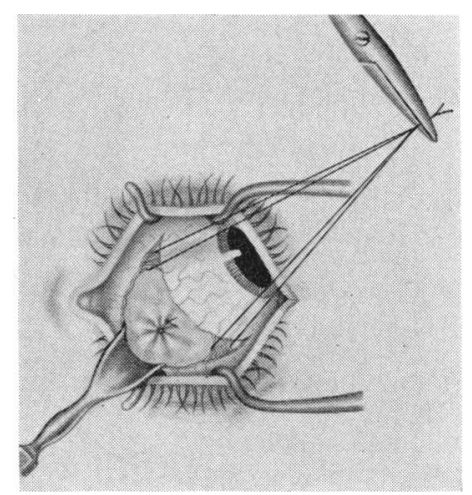

FIG. 2(d).-Disc buried and pursestring suture tightened over it. disc.

If the retinal breaks are wide apart, each break can be surrounded by a separate 
The area of the sclera to be buried need not always be circular. Any shape may be fashioned according to the size, shape, and location of the retinal break. Fig. $(3 a-d)$ shows the method used in dealing with two retinal tears situated some distance apart.

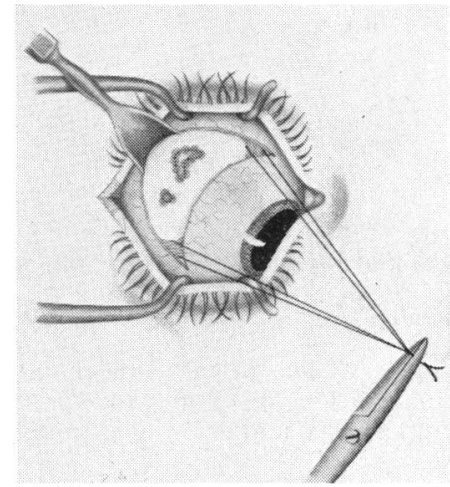

Fig. 3(a).-Diathermy applied over two adjacent retinal tears.

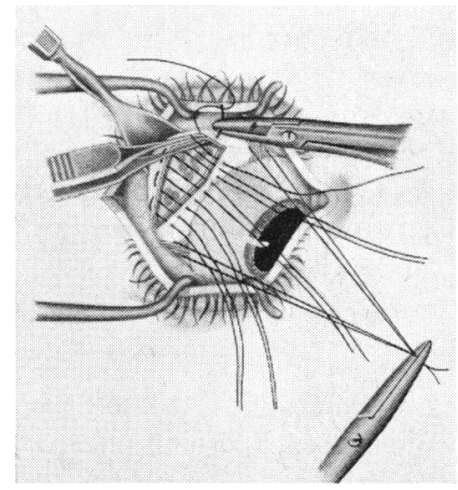

Fig. 3(c).-Mattress sutures inserted in external lip of sclera incision.

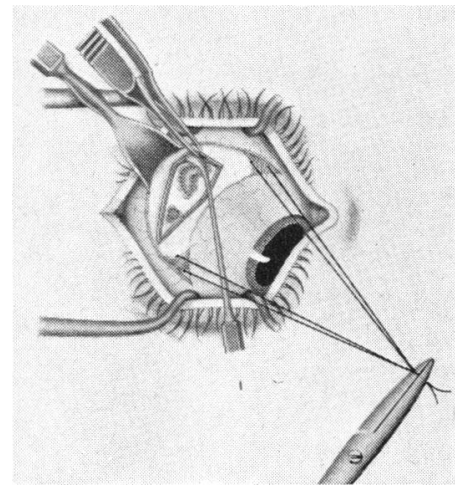

Fig. 3(b).-Scleral incision surrounding diathermy-treated area. External lip of incision undermined.

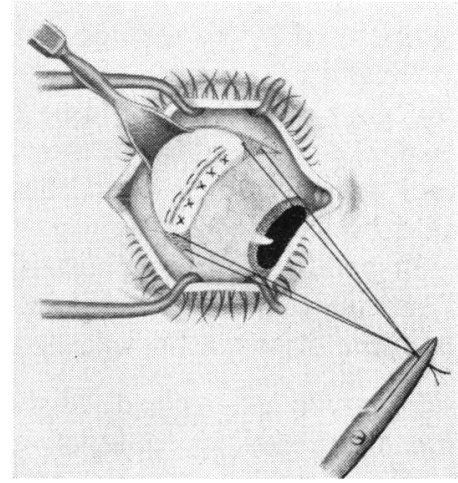

FIG. 3(d).-Sutures tied over buried sclera.

The suture material used should have high tensile strength, should not disintegrate rapidly, and should be non-irritant. Mersilene* 3/0 was found quite satisfactory. Mersilene is braided polyester fibre repeatedly cited for its superior strength and low tissue reaction. It is treated with a serum-proofing agent and is not weakened by wetting. A square knot combined with a surgeon's knot as recommended by Ethicon Limited (Fig. 4, overleaf), prevents slipping and slackening of the suture.

(5) Evacuation of Subretinal Fluid.-This should be done before the sutures are tightened. Custodis does not evacuate the subretinal fluid but relies wholly on the choroid for its resorption. He argues that the early rise in tension observed in

* Mersilene: Trade name, manufactured by Ethicon, Edinburgh, Scotland. 


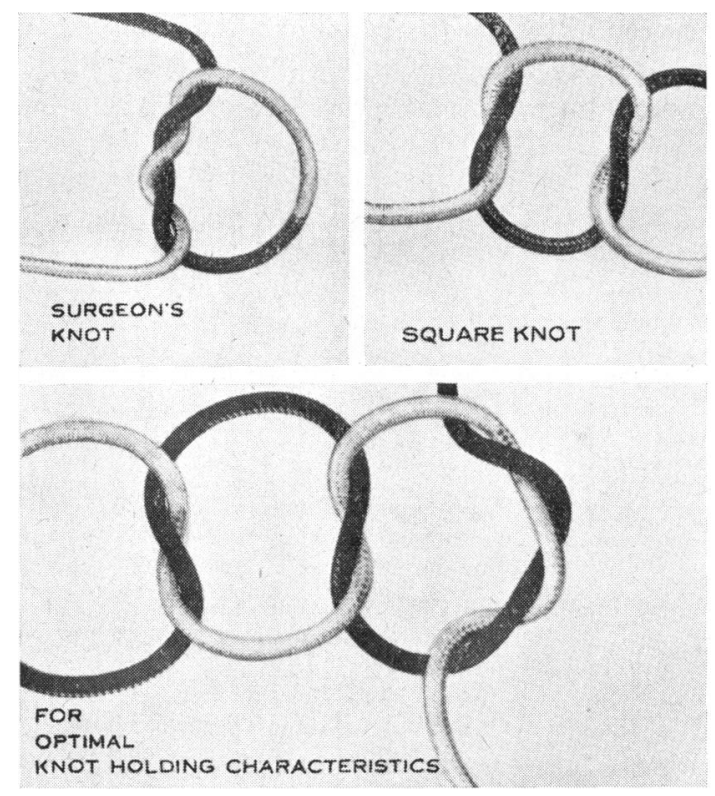

FIG. 4.-Non-slip method recommended by Ethicon Ltd. for tying Mersilene sutures (with permission).

eyes operated by his method without release of fluid helps in absorption of all the subretinal fluid. I found it extremely difficult, however, to complete my procedure of burying the sclera without evacuation. I evacuate the fluid through a tiny choroidal prick made with the diathermy needle along the posterior margin of the scleral incision. As advised by Schepens and others (1957) the diathermy needle should be held tangentially to the globe and only a tiny perforation made, thus avoiding sudden gush of fluid, injury to the retina, and vitreous loss.

The release of the subretinal fluid allows the sutures to be tightened and the outlined piece of sclera buried.

(6) Completion.-The divided rectus muscles are now re-attached to their stumps using catgut, and the conjunctiva and Tenon's capsule are stitched with interrupted fine silk sutures.

\section{Post-operative Course}

Examination of the fundus after the operation shows a white raised patch corresponding to the sclero-choroidal infold (Fig. 5a, opposite). The white colour is not due only to the diathermy coagulation since it corresponds in size to the whole buried sclera. It is probably due to choroidal anaemia produced by pressure of the buried sclera. Around this white patch the retina may still be detached if there is residual subretinal fluid. Near the centre of the white patch retinal breaks can be identified, now darker in colour than before the operation.

This appearance persists for about one week. During the second week the contrast between the rents and the retina around them gradually fades and the red colour of the choroid reappears over the buried sclera. During the third week pigmentation shows and the outline of the retinal breaks is lost, but the elevation persists for several months (Fig. 5b, opposite). 


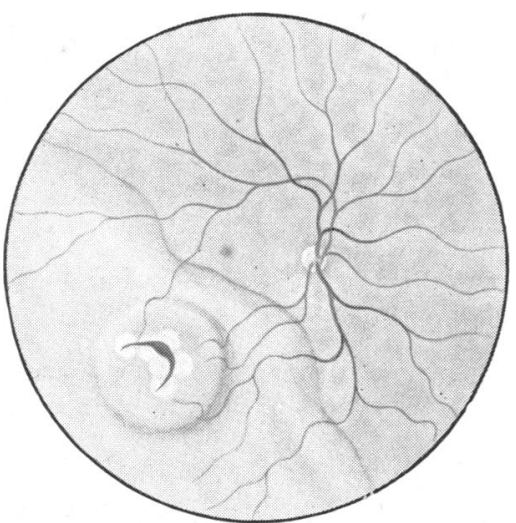

FIG. 5(a).-Circular scleral disc buried, bringing choroid closer to retina around the tear.

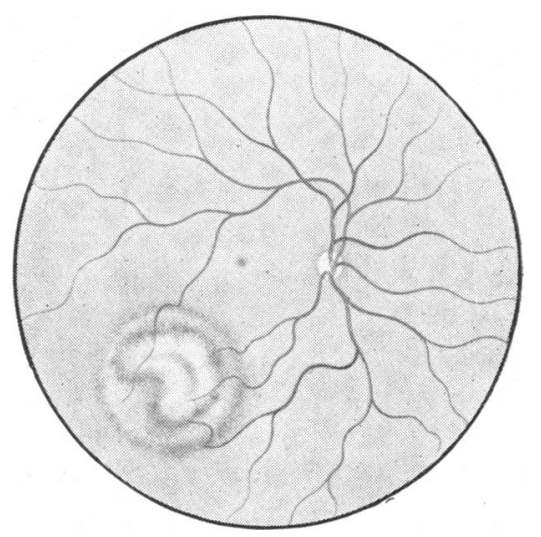

FIG. 5(b).-Appearance of fundus one month after operation.

\section{Conclusion and Summary}

(1) Subscleral scleral burial is an operation designed to bring the diathermytreated choroid closer to the retina through a wide circle around retinal breaks. This is achieved by scleral burial, which obviates the difficulties encountered in using the synthetic foreign bodies so far described to achieve the same results.

(2) The sclera which is buried is either circular or oval in shape and the choroidal infold is wide enough to cover large retinal tears. Choroidal infolds produced by synthetic implants and tubes tend to be linear and may not cover the ends of a large retinal tear.

(3) The sclera which is weakened by diathermy is completely buried, so that no weak point is left in the wall of the eyeball.

(4) This method also diminishes post-operative adhesions between Tenons capsule and sclera.

\section{REFERENCES}

BARUT, C. (1952). "Traitement du décollement de la retine par la résection sclérale lamellaire". Annequin, Lyon. (Abs. in Clinique (Paris) 1952, 47, 313).

Brockhurst, R. J., Schepens, C. L., and Okamura, I. D. (1958). A.M.A. Arch. Ophthal., 60, 1003.

Custodis, E. (1952). Ber. dtsch. ophthal. Ges., 57, 227.

(1953). Ibid., 58, 102.

(1956). Klin. Mbl. Augenheilk., 129, 476.

(1960). In "Importance of the Vitreous Body in Retina Surgery", ed. C. L. Schepens, pp. 175-181 (2nd Conference of the Retina Foundation, 1958). Mosby, St. Louis.

el BAYADI, G. (1953). Brit. J. Ophthal., 37, 625.

(1954). "Acta XVII Conc. Ophthal. 1954, Canada, U.S.A.", vol. 1, p. 100 (1961). Amer. J. Ophthal., 51, 837.

LINDNER, K. (1933). Z. Augenheilk., 81, 277.

Müller, L. (1903). Klin. Mbl. Augenheilk., 41 (2), 459.

Okamura, I. D., Schepens, S. L., and BROCKHURST, R. J. (1959). A.M.A. Arch. Ophthal., 62, 445.

Schepens, C. L., OKamura, I. D., Brockhurst, R. J., (1957). I Ibid., 58, 797.

-, , (1958). Ibid., 60, 84.

, and Regan, C. D. J. (1960). Arch. Ophthal. (Chicago), 64, 868.

ShaPland, C. DeE (1951). Trans. ophthal. Soc. U.K., 71, 29. 